(39) Nakajima, A.; Hayashi, T.; Ohmori, M. Biopolymers 1968, 6, 973.

(40) Kubo, K.; Ogino, K. Polymer 1975, 16, 629.

(41) Rai, J. W.; Miller, W. G.; Bryant, R. G. Macromolecules 1973, $6,262$.

(42) Shoij, A.; Ozaki, T.; Saito, H.; Tabeta, R.; Ando, I. Macromolecules $1984,17,1472$
(43) Czarniecka, K.; Samulski, E. T. Mol. Cryst. Liq. Cryst. 1981, $63,205$.

(44) Toriumi, H.; Matsuzama, K.; Uematsu, I. J. Chem. Phys. 1984, 81,6085 .

(45) Spiess, H. W. Adv. Polym. Sci. 1985, 66, 24.

(46) Hanabusa, H.; Sato, M.; Shirai, H.; Takemoto, K.; Iizuka, E. J. Polym. Sci., Polym. Lett. Ed. 1984, 22, 559.

\title{
Interactions between Surfaces with Adsorbed Polymers: Poor Solvent. 2. Calculations and Comparison with Experiment
}

\author{
Kevin Ingersent, ${ }^{\dagger}$ Jacob Klein,* and Philip Pincus ${ }^{\ddagger}$ \\ Magdalene College, Cambridge, England, Cavendish Laboratory, Cambridge, England, and \\ Polymer Department, Weizmann Institute of Science, Rehovot 76 100, Israel, and Corporate \\ Laboratories, Exxon Research, Clinton Township, Annandale, New Jersey 08801. \\ Received September 16, 1985
}

\begin{abstract}
Analytical expressions, based on a mean-field model and derived in a previous paper (part 1 Klein, J.; Pincus, P. A. Macromolecules 1982, 15, 1129), for the interaction between two parallel plates bearing adsorbed polymer in poor-solvent medium, are solved numerically to yield (a) segmental density profiles of the adsorbed polymer and (b) interaction-energy vs. plate-separation profiles. All parameters appearing in these calculations are obtainable from bulk data such as the polymer-solvent phase diagram and adsorbance measurements. The calculated results are critically compared with model experiments on forces between smooth mica surfaces bearing polystyrene in cyclohexane in poor-solvent conditions: the agreement is qualitatively very good and quantitatively fair. Some improvements to the model are suggested.
\end{abstract}

\section{Introduction}

It has long been known that layers of flexible polymers adsorbed at solid-liquid interfaces may strongly modify the surface-surface forces; this effect is commonly used to stabilize (or destabilize) colloidal dispersions and is also encountered in many naturally occurring biocolloidal systems. The experimental and theoretical situation up to about 1981 has been comprehensively reviewed by Vincent ${ }^{1}$ and by Vincent and Whittington. ${ }^{2}$

Theoretical treatment of the problem of interaction between surfaces bearing adsorbed polymer layers is complicated by the number of effects that may contribute; these include osmotic interactions between opposing adsorbed segments, volume-exclusion effects due to the impermeable walls, and the sticking energy of segments adsorbed on the solid surface. It may also be possible for single molecules to span the interplate gap, and such bridging always leads to attraction. In addition, any theoretical models must make assumptions about the state of equilibrium of the adsorbed polymer layers when they are moved relative to each other; as surface diffusion phenomena are often very slow, such assumptions require great care.

Over the past few years there have been a number of model experimental studies of the interaction between atomically smooth solid (mica) surfaces bearing adsorbed polymer layers and immersed in liquid media in a variety of solvency conditions. ${ }^{3-6}$ These studies are sufficiently direct to invite quantitative comparison between the experimental results and the predictions of theoretical models; in this way we may hope to get insight into the

\footnotetext{
* Cavendish Laboratory and Weizmann Institute of Science. Address correspondence to the Weizmann Institute.

${ }^{\dagger}$ Magdalene College. Present address: Department of Physics, University of Pennsylvania, Philadelphia, PA.

$\ddagger$ Exxon Research. Present address: Department of Chemical Engineering, University of California at Santa Barbara, Santa Barbara, CA 93106.
}

ways in which such models may be improved and also into the validity of the assumptions (such as state of equilibrium) made in such comparisons.

Recent attempts at analysis have fallen into two broad categories. Scheutjens and Fleer ${ }^{7}$ have applied the transfer matrix techniques of Rubin ${ }^{8}$ and DiMarzio, ${ }^{9}$ and their treatment accounts for the possible chain conformations, suitable weighted on a quasi-crystalline lattice. In the Cahn-de Gennes approach ${ }^{10-12}$ one minimizes the excess free energy of the adsorbed interacting polymer layers with respect to the segmental density profile $\phi(z)$ at distance $z$ from each surface; de Gennes used this approach to calculate the interaction-distance profile between adsorbed polymer layers in a good-solvent system, using scaling exponents to go beyond Flory-Huggins mean-field theory. ${ }^{12}$ In an earlier paper, ${ }^{13}$ hereafter referred to as part 1 , Klein and Pincus extended de Gennes' treatment ${ }^{12}$ to calculate the interaction between irreversibly adsorbed polymer layers below the $\theta$-point, where Flory-Huggins mean field is a good approximation. ${ }^{14,15}$ The assumption of irreversible adsorption is suggested by experiment ${ }^{3}$ and reflects the fact that even when the sticking energy per monomer is small, the total sticking energy per polymer molecule may greatly exceed its thermal energy $k_{\mathrm{B}} T$. Under these poor-solvent conditions an attractive interaction was predicted ${ }^{13}$ at plate separations greater than about $R_{\mathrm{g}}$ (the unperturbed radius of gyration of the polymer) and repulsive forces for narrow gaps. This agreed qualitatively with the results of direct force measurements between polystyrene (PS) layers adsorbed on mica surfaces in cyclohexane $(\mathrm{CH})$. We have now used computational methods to obtain force profiles which can be compared quantitatively with experiment.

In the next section there is a summary of the mean-field theory from part 1, leading to coupled integral equations that determine the interfacial energy. The set of coupled equations is solved numerically, as briefly described in section III, and the results are presented in section IV. In section $\mathrm{V}$ these predictions are compared critically with 


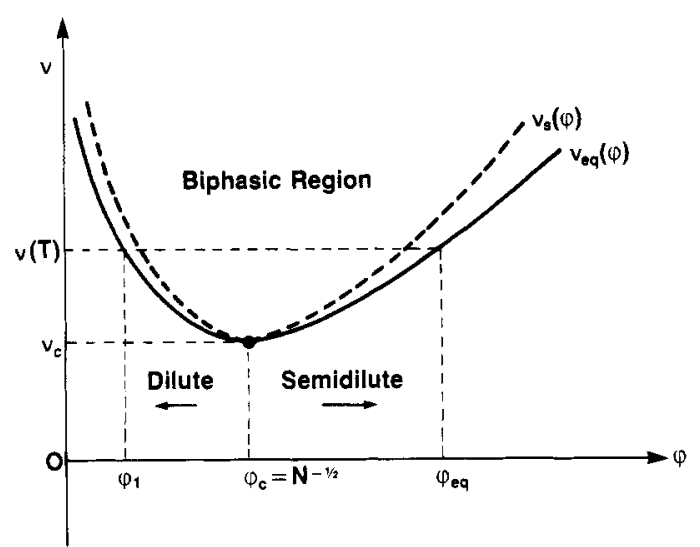

Figure 1. Flory-Huggins phase diagram for a polymer-poorsolvent system, where $v$ is the second virial coefficient and $\phi$ is the polymer volume fraction. $v_{\mathrm{s}}(\phi)$ (broken curve) and $v_{\mathrm{eq}}(\phi)$ (solid curve) are respectively the spinodal and coexistence curves separating the biphasic and one-phase regions. The critical point (at temperature $T=T_{\mathrm{c}}$ ) is at $v_{\mathrm{c}}$ and $\phi_{\mathrm{c}}$, while $\phi_{1}$ and $\phi_{\mathrm{eq}}$ are the values of $\varphi$ on the dilute and semidilute branches of the coexistence curve at $T<T_{\mathrm{c}}\left(v(T)>v_{\mathrm{c}}\right)$.

experimental results for the $\mathrm{PS} / \mathrm{CH} /$ mica system, and in section VI we make concluding remarks.

\section{Outline of the Theory}

In this section we summarize the results derived in part 1. For more details the reader is referred to that paper. ${ }^{13}$

We consider two smooth, flat plates, incubated far apart in a solution containing polymer at volume fraction $\phi_{\mathrm{b}}$ until each bears an irreversibly adsorbed surface excess of adsorbed molecules. The solution is then replaced by pure solvent, and the plates are brought to a separation $D=$ $2 h$. Provided that the movement is quasi-static, the monomer volume fraction $\phi(z)$ at all times adopts a profile in the $z$ direction, perpendicular to the surface, to minimize the total Helmholtz free energy, subject to the constraint that the surface excess remains constant.

This mean-field treatment starts from a Flory-Huggins theor $y^{14,15}$ for a monodisperse polymer solution (volume fraction of polymer $\varphi$ ) inscribed on a lattice of constant $a$. In a poor solvent the bulk free energy of mixing per site is

$$
F_{0} / T=\frac{\phi}{N} \ln \phi-\frac{1}{2} v \phi^{2}+\frac{1}{6} w \phi^{3}+\ldots
$$

(working in units where Boltzmann's constant is unity) where the first term describes the translational entropy per chain; the second and third terms can be regarded as two- and three-monomer interactions, respectively, and higher powers of $\phi$ re neglected. It is the negative coefficient of $\phi^{2}$, indicating net monomer-monomer attraction, that is responsible for the biphasic region of the phase diagram, Figure 1.

(a) Single-Plate Profile. At an interface the bulk free energy (eq II.1) is augmented by a term allowing for slow spatial variations in $\phi$ :

$$
F=F_{0}+(T / 24 \phi)(a \nabla \phi)^{2}
$$

The Cahn-de Gennes excess interfacial energy per unit area is ${ }^{11}$

$$
\gamma=\gamma_{\mathrm{s}}\left(\phi_{\mathrm{s}}\right)+\int_{0}^{\infty}\left[F / a^{3}-\mu_{\mathrm{b}} \phi+\pi_{\mathrm{b}}\right] \mathrm{d} z
$$

where $\gamma_{\mathrm{s}}\left(\phi_{\mathrm{B}}\right)$ is the surface contact energy of the first lattice layer of polymer (i.e., that in contact with the plate surface) when the volume fraction in that layer is $\phi_{s}(\equiv \phi(z=0))$ and the integrand represents the change in free energy per unit volume when a solution of volume fraction $\phi$ is formed from a bulk solution with chemical potential $\mu_{\mathrm{b}}$ and osmotic pressure $\pi_{\mathrm{b}}$. For weak adsorption the surface coverage is low, and we approximate $\gamma_{\mathrm{s}}$ by

$$
\gamma_{\mathrm{s}}\left(\phi_{\mathrm{s}}\right)=\gamma_{0}-\gamma_{1} \phi_{\mathrm{s}}
$$

The integral in (II.3) is rewritten by using the scaled variables $y=\left(\phi / \phi_{\mathrm{eq}}\right)^{1 / 2}$ and $x=z / \xi$, where $\phi_{\mathrm{eq}}$ is the polymer volume fraction on the semidilute branch of the coexistence curve in Figure 1 (for the given temperature of the experiment) and $\xi=a\left((3 / 2) v \phi_{\mathrm{eq}}\right)^{-1}$ is the Edwards mean-field correlation length ${ }^{16}$ at $\phi=\phi_{\text {eq }}$. This gives the central equation

$$
\begin{aligned}
& \left(\gamma-\gamma_{0}\right) / \gamma_{1} \phi_{\text {eq }}= \\
& -y_{\mathrm{s}}{ }^{2}+\sigma^{-1} \int_{0}^{\infty}\left[y^{6}-2 y^{4}+\beta^{2} y^{2}+\left(\frac{\mathrm{d} y}{\mathrm{~d} x}\right)^{2}\right] \mathrm{d} x
\end{aligned}
$$

The physical variables of the model are contained on the right-hand side in the surface coupling parameter

$$
\sigma=6 \gamma_{1} a \xi / T
$$

and in the parameter $\beta$, where

$$
\beta^{2}=\left[\ln \left(\phi_{\mathrm{b}} / \phi_{\mathrm{eq}}\right)+1\right] /\left(\ln \varphi_{1}\right)
$$

and where $\phi_{1}$ is the value of $\phi$ on the dilute branch of the coexistence curve, Figure 1 . We note especially that in the treatment of part 1 the translational entropy term $(\phi / N)$ $\ln \phi$ in eq II.1 was neglected when (II.5) was derived; this amounted to taking the limit of infinite chain size $(N \rightarrow$ $\infty)$ and led to an alternative expression

$$
\beta^{2}=\left(\ln \phi_{\mathrm{b}}\right) /\left(\ln \phi_{1}\right)
$$

(See Appendix for details.)

We also recall that, as shown in part 1

$$
\ln \phi_{1}=-\frac{N a^{2}}{6 \xi^{2}}=-\left(\frac{R_{\mathrm{g}}}{\xi}\right)^{2}
$$

so that the parameter $\beta$ depends on the bulk incubation concentration $\phi_{\mathrm{b}}$, the size $N$ of the polymer, and the temperature (through $\phi_{1}, \phi_{\mathrm{eq}}$ on the phase diagram, Figure 1).

The single-plate profile is found by minimizing (II.5) with respect to $y(x)$ (i.e., $y(\phi(x))$ and $y_{\mathrm{s}}\left(\equiv y(0)=\left(\phi_{\mathrm{s}}\right)\right.$ $\left.\left.\phi_{\mathrm{eq}}\right)^{1 / 2}\right)$ )

$$
\left(\frac{\mathrm{d} y}{\mathrm{~d} x}\right)^{2}=y^{6}-2 y^{4}+\beta^{2} y^{2}
$$

and, for $\sigma>\left(\beta^{2}-1\right)^{1 / 2}$, we find

$$
y_{\mathrm{s}}{ }^{2} \equiv \phi_{\mathrm{s}} / \phi_{\mathrm{eq}} \simeq \sigma+1
$$

The proximal part of the monomer profile $\phi(z)$ is always in the semidilute concentration regime of the phase diagram $\left(y_{\mathrm{s}}^{2}>1\right)$, while for $\beta>1$ the distal part of the profile is in the dilute regime. Therefore $\phi(z)$ passes through the unstable biphasic regime $\left(\phi_{\mathrm{eq}}>\phi(z)>\phi_{1}\right.$, Figure 1). The surface excess of polymer per unit area $\Gamma$ is given by

$$
\begin{aligned}
\Gamma a^{2} & =a^{-1} \int_{0}^{\infty}\left[\phi(z)-\phi_{\mathrm{b}}\right] \mathrm{d} z \\
& =1 / 2 w^{-1 / 2} \ln [2 \sigma /(\beta-1)]
\end{aligned}
$$

(b) Two-Plate Profile. When two plates, after incubation a long way apart to a surface excess $\Gamma$ (eq II.10), are washed (i.e., polymer solution is replaced by pure solvent) and brought together, the surface excess $\Gamma$ may be held constant by the introduction of a Lagrange multiplier $k$, which we call (following de Gennes ${ }^{12}$ ) the pseudochemical potential. We write an augmented interfacial 
energy density $\tilde{\gamma}$, given, in analogy to (II.5), by

$$
\left(\frac{\tilde{\gamma}-\gamma_{0}}{\gamma_{1} \phi_{\mathrm{eq}}}\right)=-y_{\mathrm{s}}{ }^{2}+\sigma^{-1} \int_{0}^{h / \xi}\left[G(y)+\left(\frac{\mathrm{d} y}{\mathrm{~d} x}\right)^{2}\right] \mathrm{d} x
$$

where $G(y)=y^{6}-2 y^{4}+k y^{2}$.

Minimizing with respect to $y(x)$ and ensuring that the profile is symmetric about the midpoint, where $y(h / \xi)=$ $y_{m}$, we find

$$
(\mathrm{d} y / \mathrm{d} x)^{2}=G(y)-G\left(y_{\mathrm{m}}\right)
$$

which may be integrated to give

$$
h / \xi=\int_{y_{\mathrm{m}}}^{y_{\mathrm{s}}}\left[G(y)-G\left(y_{\mathrm{m}}\right)\right]^{-1 / 2} \mathrm{~d} y
$$

Finally, minimizing $\tilde{\gamma}$ (eq II.11) with respect to $y_{\mathrm{s}}$ yields

$$
\sigma y_{\mathrm{s}}=\left[G\left(y_{\mathrm{s}}\right)-G\left(y_{\mathrm{m}}\right)\right]^{1 / 2}
$$

The third equation for $y_{\mathrm{s}}, y_{\mathrm{m}}$, and $k$, and thus the profile, is the constraint on the surface excess $\Gamma$

$$
\begin{aligned}
\Gamma a^{2} & =a^{-1} \int_{0}^{h} \phi(z) \mathrm{d} z \\
& =w^{-1 / 2} \int_{0}^{h / \xi} y^{2} \mathrm{~d} x= \\
& =1 / 2 w^{-1 / 2} \int_{y_{\mathrm{m}}}^{y_{\mathrm{s}}} y^{2}\left[G(y)-G\left(y_{\mathrm{m}}\right)\right]^{-1 / 2} \mathrm{~d} y
\end{aligned}
$$

from (II.10).

The interplate pressure is

$$
\pi(h)=-\partial \tilde{\gamma} / \partial h=-G\left(y_{\mathrm{m}}\right) T /\left(6 w^{1 / 2} \xi^{3}\right)
$$

The quantity measured experimentally in the force studies is the interfacial energy per unit area between two flat plates a distance $D$ apart. This is obtained in our model (for each plate) by integration

$$
\begin{aligned}
\gamma(D)=\int_{\infty}^{h=D / 2} \pi(z) \mathrm{d} z & = \\
& -T /\left(6 w^{1 / 2} \xi^{2}\right) \int_{\infty}^{h / \xi} G\left[y_{\mathrm{m}}(z)\right] \mathrm{d} z
\end{aligned}
$$

The set of coupled equations II.13-II.15 has analytic solutions only in the special cases $h \rightarrow 0, h \rightarrow \infty$, and $h=$ $h_{\text {eq }}$, where $\pi\left(h_{\text {eq }}\right)=0$; these are presented in part $1 .{ }^{13}$ For general separations numerical methods are required to calculate $y_{s}, y_{m}$, and $k$, which then fix the entire profile and in turn permit the evaluation of $\pi(h)$ and $\gamma(D)$. In section III we describe briefly how this is done, and in the following sections we present general features of the results and their comparison with the surface force experiments.

\section{Method of Computation}

The problem of solving the coupled equations II.13-II.15 can be reduced to the pair of simultaneous nonlinear equations

$$
\begin{gathered}
\tilde{A}\left(y_{\mathrm{m}}, y_{\mathrm{s}}\right) \equiv \int_{y_{\mathrm{m}}}^{y_{\mathrm{s}}}\left[G(y)-G\left(y_{\mathrm{m}}\right)\right]^{-1 / 2} \mathrm{~d} y=A \\
\tilde{B}\left(y_{\mathrm{m}}, y_{\mathrm{s}}\right) \equiv \int_{y_{\mathrm{m}}}^{y_{\mathrm{s}}} y^{2}\left[G(y)-G\left(y_{\mathrm{m}}\right)\right]^{-1 / 2} \mathrm{~d} y=B
\end{gathered}
$$

where $A=h / \xi$ and $B=1 / 2 \ln [2 \sigma /(\beta-1)]$ are constants. The pseudochemical potential $k$, required for evaluation of $G(y)$, is obtained explicitly in terms of $y_{m}$ and $y_{s}$ by inverting (II.14). The best estimate of $y_{\mathrm{m}}$ and $y_{\mathrm{s}}$ was obtained by minimizing a combined measure of error $\left[\tilde{A}\left(y_{\mathrm{m}}, y_{\mathrm{s}}\right)-A\right]^{2}+c\left[\tilde{B}\left(y_{\mathrm{m}}, y_{\mathrm{s}}\right)-B\right]^{2}$. Generally $A$ and $B$ are similar in magnitude, so setting $c$ to unity ensures satisfactory convergence.

Solutions for $y_{\mathrm{m}}$ and $y_{\mathrm{s}}$ and hence the osmotic pressure $\pi$ were calculated for successively smaller plate separations $D$, starting from some separation $D_{\max }$ where $\pi\left(D_{\max }\right)$ was less than $0.5 \%$ of the maximum pressure. Numerical quadrature of the osmotic pressure between $D_{\max }$ and $D$ then yielded an estimate of the interation energy per unit area $\gamma(D)$ to better than $5 \%$.

Segmental density profiles $\phi(z) \equiv \phi_{\text {eq }} y^{2}(x)$ were calculated by integration of the gradient $\mathrm{d} y / \mathrm{d} x$, given by (II.8) for a single plate and by (II.12) between two plates, starting at the surface $(x=0)$ where $y=y_{\mathrm{s}}$ is known.

\section{Computed Results}

We present results for the polymer segment density profile $\phi(z)$ and the adsorbance $\Gamma$; for the two-plate situation we present in addition the interaction-distance profile, in terms of $\gamma(h)$, which is related to the force profiles measured experimentally. The results are, as far as possible, scaled so that they depend only on $\beta$ (containing in it the temperature, incubation concentration, and polymer size) and the surface coupling parameter $\sigma$.

Values of $\beta$ were chosen in a range consistent with experimental data, ${ }^{3}$ effectively $1 \leq \beta \leq 2$ (see also section V). Fixing $\sigma=6 \gamma_{1} a \xi / T$ (eq II.6) is complicated by the absence of direct measurements of $\gamma_{1}$ (the net adhesive energy per unit polymer volume at the surface). In principle, it is possible to fix $\sigma$ by experimentally measuring the adsorbance $\Gamma$, related to $\sigma$ by eq II.10. In the present treatment we choose values of $\sigma$ that satisfy all the inequalities necessary for the theory of part 1 to be valid: (a) weak adsorption, so that $\gamma_{\mathrm{s}}\left(\phi_{\mathrm{s}}\right)$ is linear in $\phi_{\mathrm{s}}$

$$
\gamma_{1} a^{2} / T \ll 1 \text {, or } \sigma \ll 6 \xi / a=6 / w^{1 / 2} \phi_{\mathrm{eq}}
$$

For the single-plate case, $\phi_{\mathrm{s}} \ll 1$ or $\sigma \ll 1 / \phi_{\text {eq }}-1$. (b) irreversible adsorption, $N\left(\gamma_{1} a^{2} / T\right)^{2} \gg 1$ or $\sigma \gg 6 \xi /\left(N^{1 / 2} a\right)$ $=\left[-6 / \ln \phi_{1}\right]^{1 / 2}$. (c) strong coupling, $\sigma \gg 1$ and $\sigma \gg\left(\beta^{2}\right.$ $-1)^{1 / 2}$.

In the $\mathrm{PS} / \mathrm{CH} /$ mica system at $24^{\circ} \mathrm{C}(297 \mathrm{~K})$ the values of $\phi_{\mathrm{eq}}$ and $\phi_{1}$ are ca. 0.2 and $1.5 \pm 0.5 \times 10^{-4}$, respectively. ${ }^{13}$ The third virial coefficient $w=0.2 \pm 0.1^{17}$ or $w=0.28^{18}$; in this paper we use $w=0.2$. Combining the above conditions then gives $1<\sigma<4$, which is partially satisfied for $\sigma=2$ or 3 . Since the upper limit, set by the expression $1 / \phi_{\text {eq }}-1$, is sensitive to small changes in $\phi_{\text {eq }}$, results were also computed for other $\sigma$ values to investigate trends as the surface coupling increases.

(1) Single-Plate Results. Figure 2 shows the equilibrium polymer profile $\phi(z)$ for a single plate incubated in polymer solution for several values of $\beta$. The axes are normalized as $\phi / \phi_{\text {eq }}$ vs. $z / \xi$. The profile falls rapidly in the proximal region $z \leqslant \xi$, where $\phi$ depends almost entirely on the surface coupling constant and very little on the bulk conditions. In contrast, the central and distal regions of the profile depend strongly on $\beta$. It can also be readily shown (for example, from eq II.8) that for $\beta^{2}<9 / 8$ a point of inflexion is expected in the $\phi(z)$ profile; indeed at low $\beta$ values $\left(\beta^{2}<9 / 8\right)$ the profiles show a broad plateau where the polymer crosses the unstable part of the bulk-phase diagram $\left(\phi_{\mathrm{u}}<\phi<\phi_{\mathrm{eq}}\right)$. In the tail $(z \gtrsim 3 \xi), \phi$ falls off exponentially with decay length $\xi / 2 \beta$, as also calculated in part 1 (this is more clearly seen on a $\log \phi$ vs. $z$ plot of the data, inset to Figure 2).

In Figure 3 the surface excess $\Gamma$ is plotted (solid curve) (normalized as $w^{1 / 2} \Gamma a^{2}$ ) against bulk incubation concentration $\phi_{\mathrm{b}}$ for a system with $\phi_{\mathrm{eq}}=0.2$ and $\phi_{1}=1.5 \times 10^{-4}$ (similar to the PS/cyclohexane system at $24^{\circ} \mathrm{C}$ for $M=$ $6 \times 10^{5}$ polystyrene). There is a weak (logarithmic) de- 


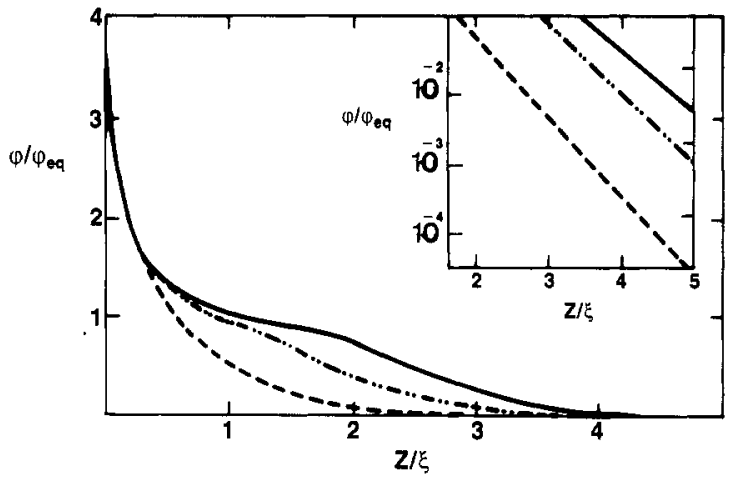

Figure 2. Calculated segmental density profiles $\phi(z)$ near an adsorbing wall $(z=0)$, normalized with respect to $\phi_{\mathrm{eq}}$ and the Edwards correlation length $\xi$ for different values of $\beta$ : $(-) \beta=$ $1.005 ;(\ldots) \beta=1.04 ;(\cdots) \beta=1.16$. The inset shows the exponential decay of $\phi(z)$ in the tail region of the adsorbed layers. The exponential decay length is in each case equal, within error, to $\xi / 2 \beta$. (See also text.)

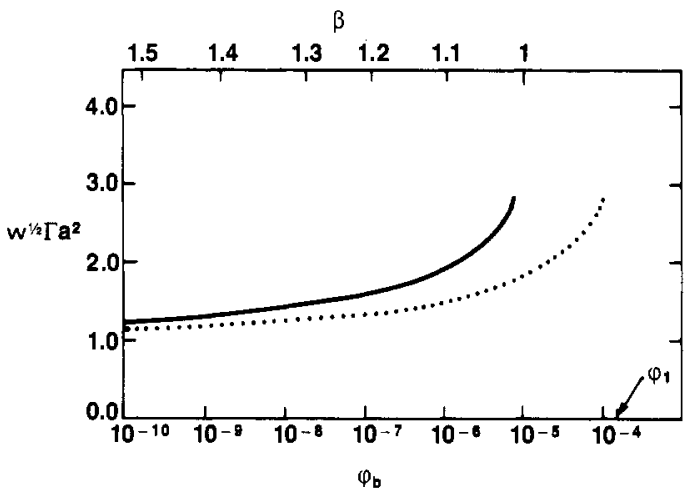

Figure 3. Calculated surface excess $\Gamma$ (normalized as $w^{1 / 2} \Gamma a^{2}$ ) as a function of bulk incubation volume fraction $\phi_{\mathrm{b}}$. The top axis gives the corresponding value of $\beta$ from eq $\Pi .7$, and the solid curve is the calculated surface excess based on this definition of $\beta$. The dotted curve is calculated on the basis of the definition of $\beta$ used in part $1 .^{13}$ (See also text.)

pendence of $\Gamma$ on $\phi_{\mathrm{b}}$ for $\phi_{\mathrm{b}} \lesssim 0.01 \phi_{1}$ and a rapid rise in $\Gamma$ as the bulk concentration approaches $\phi_{1}$ (the coexistence concentration). Using the new renormalized form of $\beta$ (eq II.7), we find that this sharp rise in the adsorbance begins at lower values of $\phi_{\mathrm{b}}$ than originally suggested in part 1. This is illustrated in Figure 3. Experiments are currently in preparation (with $\mathrm{H}$. Terashima ${ }^{19}$ ) to check the form of $\Gamma\left(\phi_{\mathrm{b}}\right)$ directly.

(2) Two-Plate Results. In Figures 4 and 5, reduced surface interaction energies per unit area (of each plate), $\gamma_{\mathrm{red}}=\gamma / \gamma_{\mathrm{sc}}$, where $\gamma_{\mathrm{sc}}=k_{\mathrm{B}} T /\left(6 w^{1 / 2} \xi^{2}\right)$, are plotted against plate separation $D$, scaled as $D / \xi$. In every case the general shape of the curve exhibits the same features: strong repulsion between the plates for small separations, the existence of an equilibrium position at $D=D_{\text {eq }}$ where the osmotic pressure vanishes and $\gamma=\gamma_{\min }$ is a minimum, and attraction for wider gaps.

In Figure 4 the incubation parameter $\beta$ is varied at fixed $\sigma$; two trends appear as $\beta$ increases ( $\phi_{b}$ decreases for a given polymer size and ambient temperature):

(a) The $D$ scale is compressed, consistent with an interaction induced by overlap of two adsorbed layers, each having an effective thickness

$$
\begin{aligned}
d & =\frac{\Gamma a^{3}}{\phi_{\mathrm{eq}}}=\frac{\xi}{2} \ln [2 \sigma /(\beta-1)] \\
& =\frac{\beta R_{\mathrm{g}}}{2\left(\ln \phi_{\mathrm{b}}^{-1}\right)^{1 / 2}} \ln [2 \sigma /(\beta-1)]
\end{aligned}
$$

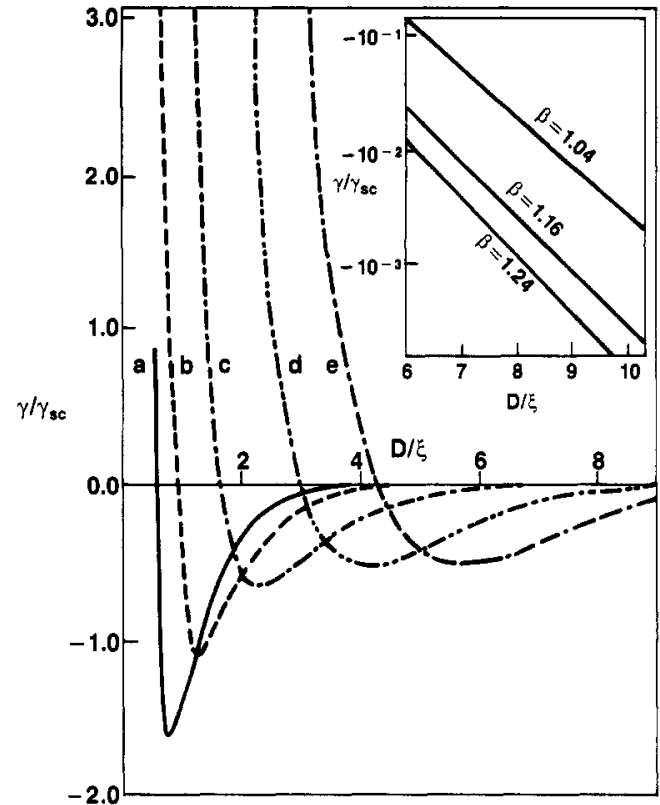

Figure 4. Calculated interaction energy $\gamma$ per unit area (of each plate) for two flat parallel plates a distance $D$ apart, following irreversible adsorption of polymer from solution, for $\sigma=3$ and different values of $\beta$. Interaction and distance scales are normalized with respect to $\gamma_{\mathrm{sc}}$ and $\xi$ : (a) $\beta=1.90$; (b) $\beta=1.50$; (c) $\beta=1.16$; (d) $\beta=1.025$; (e) $\beta=1.005$. The inset shows the exponential decay of $\gamma$ in the tail region of the interaction for three values of $\beta . \quad(\gamma<0$ corresponds to attractive interaction energies.)

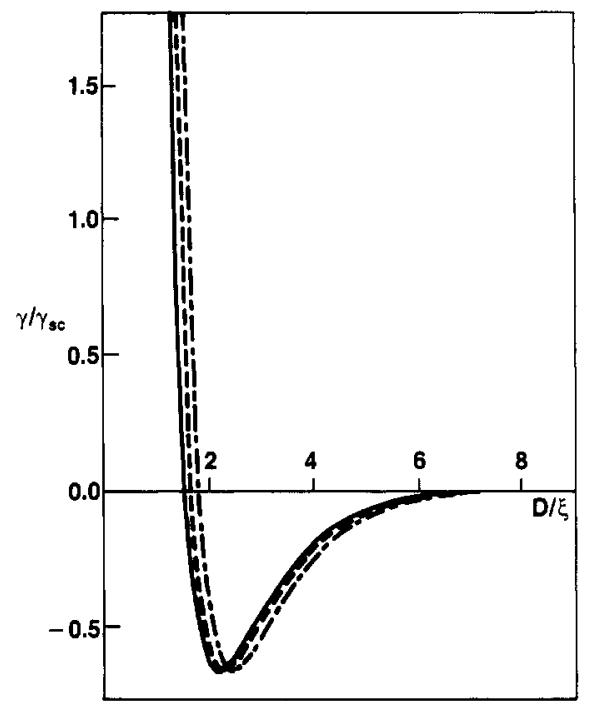

Figure 5. Interaction-distance profiles (axes as Figure 4) for $\beta$ $=1.16$ and different values of the surface coupling parameter $\sigma$ : $(-) \sigma=2 ;(\cdots) \sigma=3 ;(--) \sigma=8$.

where $R_{\mathrm{g}}$ is the unperturbed radius of gyration of the polymer.

(b) The strength of the interaction (i.e., $\left.\left|\gamma_{\min }\right|\right)$ increases. Now it is found (see also Figure 2 and Figure 6) that $\phi(z)$ decreases, with decreasing $\phi_{\mathrm{b}}$ or increasing $\beta$, at all $z$ values; this would be expected to decrease the magnitude of both (a) the sticking energy (which is proportional to $\phi_{\mathrm{s}}=\phi(0)$ ) and (b) the osmotic attraction (going as $\phi^{2}$ ) in eq II.11. Therefore the observed trend (of increasing $\left.\left|\gamma_{\min }\right|\right)$ requires that the decrease (at higher $\beta$ ) in the gradient term and the pseudochemical potential terms in the expression for $\tilde{\gamma}$ (eq II.11) more than compensates for the other two effects $a$ and $b$. In other words, at lower adsorbance of polymer on the surface the increase in free energy associated with compressing the two plates and the irreversibly adsorbed polymer between them (which would like to 


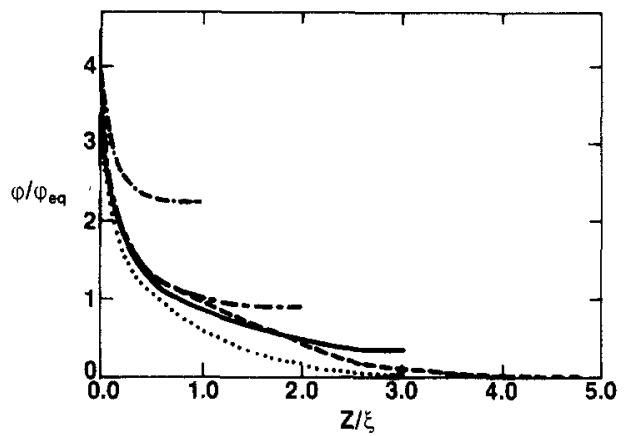

Figure 6. Adsorbed polymer segment concentration vs. distance profile (at fixed surface excess $\Gamma$ ) between two plates at different plate separations, plotted as normalized volume fraction $\phi / \phi_{\text {eq }}$ against scaled distance $z / \xi$ from one of the plates. Profiles are symmetrical about the end point of each curve; the plates separation $D$ is twice the value of $z$ at each end point, i.e., for $\beta=1.04$ and $(-\cdots) D=2 \xi,(\cdots) D=4 \xi,(-) D=6 \xi,(--) D=10 \xi$, and for $\beta=1.16(\cdots) D=6 \xi$

escape in full equilibrium) is low; the net attractive contribution due to the osmotic attraction and sticking energy (including bridging) is then greater. This may seem contrary to intuition and emphasizes the restricted-equilibrium nature of the situation.

When $\beta$ is held contant but $\sigma$ is increased, there is a small expansion of the $D$ scale (Figure 5 ), as expected from the $\sigma$ dependence of the effective layer thickness $d$ in eq IV.1). Over the range of $\sigma$ used in these calculations (2 $\leq \sigma \leq 8$ ), there is no significant vartiation in the depth of the attractive well, $\left|\gamma_{\min }\right|$, indicating almost complete cancellation between the three effects described above: sticking energy and osmotic attraction, on the one hand, and repulsion due to irreversibly adsorbed polymer on the other (where increasing $\sigma$ and reducing $\beta$ produce similar trends).

For separations $D$ greater than about $6 \xi$ the energy profile has an exponential tail (inset to Figure 4). When $\sigma=3$ the decay length increases from ca. $0.4 \xi$ to $0.5 \xi$ in the range $\beta=1.04-1.24$.

Figure 6 shows monomer profiles $\phi / \phi_{\mathrm{eq}}$ vs. $z / \xi$ between two plates for different separations $D$. With $D$ as low as $2 \xi$ the volume fraction falls rapidly away from each surface and the greater part of the gap is occupied by a slowly varying polymer concentration that lies in the unstable part of the bulk phase diagram $\left(\phi_{1}<\phi<\phi_{\text {eq }}\right.$, Figure 1). The profiles also show that the monomer density $\phi(z)$ at any point well away from the plates does not always fall as $D$ increases (e.g., at $z=\xi$ ) and that in addition a plateau-like region may form, similar to the single-plate case (Figure 2), for $D \gtrsim 10 \xi$. Also shown in Figure 6 (dotted line) is the $\phi / \phi_{\text {eq }}$ profile for a larger $\beta$ value, for $D=6 \xi$, illustrating that in such a case (corresponding to lower $\phi_{\mathrm{b}}$ and adsorbance of polymer) $\phi(z)$ is indeed lower at all $z$ values, as noted earlier. The occurrence of volume fractions $\phi>1$ very close to the surface will be discussed in section $\mathrm{V}$.

As a control, we also compared the analytic predictions of part 1 in the limits $h$ (or $z$ for the single plate) $\rightarrow 0, h$ $\rightarrow \infty$, and $h=h_{\text {eq }}$ (where $\pi\left(h_{\mathrm{eq}}\right)=0$ or $\left.\tilde{\gamma}=\tilde{\gamma}_{\min }\right)$ with the present calculations. These included the following: (a) the proximal concentration profile for adsorption onto a single plate, given by

$$
\frac{\phi}{\phi_{\mathrm{eq}}}=\frac{\sigma+1}{1+z / D}, \quad D=\xi / 2 \sigma
$$

(b) the exponential decay of $\phi$ in the distal region of the adsorbed layer (single plate, see also inset to Figure 2)

$$
\phi \propto e^{-z / \alpha}, \quad \text { where } \alpha=\xi / 2 \beta
$$

(c) the equilibrium half separation $h_{\mathrm{eq}}$ for the two-plate case

$$
h_{\mathrm{eq}}=\frac{\xi}{2 \beta(2 / \beta-1)^{1 / 2}} \ln \left\{\frac{(\beta-1)}{1-[\beta(2-\beta)]^{1 / 2}} \frac{\sigma}{(\sigma+1)}\right\}
$$

(d) the variation

$$
\pi(h) \propto h^{-P}
$$

with $P \rightarrow 3$ as $h \rightarrow 0$ for the two-plate case.

In cases $a-c$ the calculated values were, within error, identical with the analytical values in these limits, while in case $\mathrm{d}$ the exponent closely approached $P=3$ at the lowest $h$ values considered.

\section{Comparison with Experiment}

The numerical solutions of the previous section broadly satisfy the assumptions of part $1^{13}$ of this series with regard to the various length scales; in particular, it is possible, over a restricted range of $\sigma$, to model weak but irreversible adsorption and strong surface coupling. The experiments we compared with are those in ref 3 and 5 , polystyrene (PS) in cyclohexane (CH) in poor-solvent conditions adsorbed onto mica surfaces, where these conditions appear to apply.

We first examine the interaction energy profile $\gamma(D)$ and the adsorbance $\Gamma$ for the PS $/ \mathrm{CH} /$ mica system at $24^{\circ} \mathrm{C}$. In ref 3 the polystyrene sample PS 1 had molecular weight $M=6 \times 10^{5}$, unperturbed radius of gyration $R_{\mathrm{g}}=21 \mathrm{~nm}$, incubation concentration $7 \pm 2 \times 10^{-6} \mathrm{~g} \mathrm{~mL}^{-1}$, corresponding to $\phi_{\mathrm{b}}=6.5 \pm 2 \times 10^{-6}$ when account is taken of partial specific volume, ${ }^{20}$ and temperature $T=297 \mathrm{~K}$. The value of $\phi_{\text {eq }}$ for this system is ca. $0.2,{ }^{21}$ while $\phi_{1}$ is ${ }^{13}$ ca. 1.5 $\times 10^{-4}$; we take the third coefficient $w=0.2^{17}$ and the surface coupling parameter $\sigma=3$, as discussed in the previous section (changes in the range about $\sigma=3$ make little difference, see Figure 5). From the expressions following eq II.7 the Edwards correlation length is now determined to be $\xi=7.1 \pm 0.2 \mathrm{~nm}$.

When the definition of section II (eq II. 7 is used), $\beta=$ $1.033_{-0.015}^{+0.02}$, where the range is due to uncertainty in $\phi_{\mathrm{b}}\left(\phi_{1}\right.$ being taken at its median value $\phi_{1}=1.5 \times 10^{-4}$ and $\phi_{\mathrm{eq}}=$ 0.2 ). If we neglect the translational entropy term, as in part 1, we obtain $\beta=1.16 \pm 0.2$; whenever the result depends on $\beta$, the value for $\beta=1.03$ will be followed, in square brackets, by that for $\beta=1.16$.

Figure 7 reproduces the $(F / R)$ vs. $\left(D / R_{\mathrm{g}}\right)$ profiles for samples PS1 and PS2 $\left(M=10^{5}\right)$ from ref 3 . In this experiment the force $F$ between crossed cylindrical mica surfaces, radius of curvature $R$, was measured and converted to the interfacial energy $E(D)$ between unit areas between two flat parallel plates a distance $D$ apart by using the Derjaguin approximation

$$
F(D) / 2 \pi R=E(D)=2 \gamma(D)
$$

where the factor 2 appears because $\gamma(D)$ is in these calculations the surface energy per plate.

Both curves show the same features as the computed plots in Figure 4: strong repulsion for low plate separations, a position of zero force between the curved surfaces, and attraction for wider gaps. The experimental equilibrium separation (i.e., at $F / R$ a minimum) for PS1 was

$$
D_{\text {eq }}=(1.15 \pm 0.1) R_{\mathrm{g}}
$$

close to the computed

$$
D_{\text {eq }}=\left(1.3_{-0.1}^{+0.3}\right) R_{\mathrm{g}} \quad\left[(0.8 \pm 0.1) R_{\mathrm{g}}\right]
$$




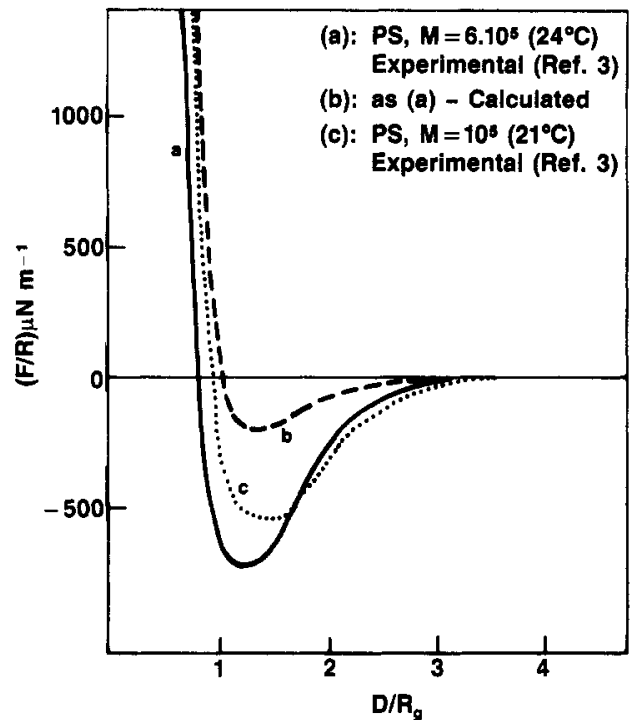

Figure 7. Interaction-distance profiles between mica sheets following adsorption of polystyrene in cyclohexane, taken from ref 3 (curves a and c) and calculated (curve b): (a) $M=6 \times 10^{5}$ $\left(24^{\circ} \mathrm{C}\right.$ ), experimental; (b) as (a), calculated by using $\beta=1.03$ (see text); (c) $M=10^{5}\left(21^{\circ} \mathrm{C}\right)$, experimental.

The range of interaction can be compared by evaluating the reduced exponential decay "length"

$$
\delta=D_{1} / D_{\text {eq }}
$$

where $\gamma\left(D_{1}\right)=\gamma_{\min } e^{-1}$; experimentally

$$
\delta=1.6 \pm 0.2
$$

in good agreement with the calculated value

$$
\delta=1.6 \pm 0.1 \quad[1.7 \pm 0.1]
$$

The theory thus appears to describe satisfactorily the spatial aspects of the interaction; however, there is a factor of 3-4 underestimate of the strength of interaction: we find

$$
2\left|\gamma_{\min }\right| \simeq 120 \mu \mathrm{J} \mathrm{m}^{-1} \quad \text { (experimental, ref 3) }
$$

while (with $\gamma_{\mathrm{sc}}=31 \mu \mathrm{J} \mathrm{m}^{-2}$ ) Figure 4 gives

$$
2\left|\gamma_{\min }\right|=31 \mu \mathrm{J} \mathrm{m}^{-2} \quad\left[40 \mu \mathrm{J} \mathrm{m}^{-2}\right]
$$

The calculated profile $(2 \gamma(D))$ for $\beta=1.03$ is drawn in on Figure 7 for comparison (curve b).

The results for PS2 (Figure 7, curve c) taken at 21-22 ${ }^{\circ} \mathrm{C}$ cannot be compared quantitatively with our calculations in the same way as for PS1, because $v$ and $\phi_{1}$, in particular, are strongly temperature dependent and vary rapidly near the critical temperature $T_{c}^{14}$ (where $\phi_{1}=\phi_{\mathrm{eq}}$, see also Figure 1), which is $30^{\circ} \mathrm{C}$ for PS1 and $25^{\circ} \mathrm{C}$ for PS2. ${ }^{22}$

The mass $m$ of polymer adsorbed per unit area of mica at equilibrium was estimated for PS1 (at $24^{\circ} \mathrm{C}$ ) from refractive index measurements ${ }^{3}$ and also (at $34^{\circ} \mathrm{C}$ ) by direct microbalance measurements. ${ }^{22}$ It is simple to show that

$$
m=M \Gamma a^{2} / 6 N_{\mathrm{A}} R_{\mathrm{g}}{ }^{2}
$$

where $N_{\mathrm{A}}$ is Avogadro's number. Using this expression, we calculate

$$
m=2.3 \pm 0.2 \mathrm{mg} \mathrm{m}^{-2} \quad\left[1.5 \pm 0.1 \mathrm{mg} \mathrm{m}^{-2}\right]
$$

compared with the measured ${ }^{3,23}$

$$
m=5-6 \mathrm{mg} \mathrm{m}^{-2}
$$

While there is some uncertainty in the experimentally determined values, the discrepancy appears too large to be due to such ambiguity.
At this point we note two shortcomings of our model, which may have bearing on the above observations. First, the real volume of monomers in the solution has been neglected. A van der Waals type correction ${ }^{23}$ would be the replacement of (II.1) by an equation of the form

$$
\frac{F_{0}}{T}=\frac{\phi}{N} \ln \left(\frac{\phi}{1-\phi}\right)-\frac{1}{2} v\left(\frac{\phi}{1-\phi}\right)^{2}+\frac{1}{6} w\left(\frac{\phi}{1-\phi}\right)^{3}
$$

While complicating the subsequent algebra of section II, this modification would increase the interplate repulsion for $D<D_{\text {eq. }}$. It would also tend to suppress the occurrence, at low plate separations, of surface volume fractions greater than unity, as obtained when (II.1) is used.

Secondly, it is found ${ }^{24}$ that for the PS/CH system the polymer-solvent interaction parameter $\chi$ increases markedly with concentration. Since $v$ in eq II.1 (or eq V.2) goes as ${ }^{14,15}$

$$
v \sim(2 \chi-1)
$$

we have that it also increases with $\phi$ for the PS/CH system. As the second virial coefficient is taken as constant in our model, this actual increase of $v$ with polymer volume fraction (in eq II.1) must lead to a decrease in the effective value of $w$ at high $\phi$. The determination of $w$ in ref 17 , employing surface tension measurements at a repulsive interface (where $\phi$ is necessarily low) under $\theta$ conditions, may thus have yielded a figure that is inapplicable to the case we are studying. If $w$ were lower, there would be an increase in the predicted surface excess (Figure 3 ) and in the strength of interaction (through an increase in $\gamma_{\mathrm{sc}}$ ), both of which would lead to closer agreement with experiment.

The effect on the interaction profile of varying the surface excess $\Gamma$ at $T<\theta$ in the PS/CH/mica system has also been investigated. In ref 5 low surface coverage was achieved as follows: the mica surfaces were kept close together while polymer was added to the solvent, thus creating a narrow gap into which polymer had to diffuse from the bulk solution before adsorbing onto the region of interest. The rate of adsorbance was thus severely diffusion-limited, and a low value of the adsorbance, $\Gamma=$ $\Gamma^{\prime}$, could be obtained, representative of the effective local polymer concentration $\phi_{\mathrm{b}}{ }^{\prime}$ in the gap; this local concentration $\phi_{\mathrm{b}}{ }^{\prime}$ is considerably lower than $\phi_{\mathrm{b}}$, the bulk polymer concentration, and it was found that $\Gamma^{\prime}$ is effectively constant over times long compared with the force measurements if the gap is kept narrow. ${ }^{5}$ To establish the full adsorbance $\Gamma$ characteristic of the bulk concentration $\phi_{b}$, the mica sheets were taken a long way apart and incubated overnight. (A fuller discussion of this experimental approach is given in ref 6 .)

Now (for a given system) a lower surface excess, characteristic of a lower effective incubation concentration, corresponds to higher $\beta$ values (Figure 3). From the computed results in Figure 4 we expect a higher $\beta$ value to lead to a shorter-ranged but stronger interaction. This is indeed shown in Figure 8, which reproduces the results from ref 5 for polystyrene, $M=9 \times 10^{5}$, at the two surface excess values $\Gamma^{\prime}$ (ca. $1.1 \mathrm{mg} \mathrm{m}^{-2}$ ) and $\Gamma$ (ca. $3.6 \mathrm{mg} \mathrm{m}^{-2}$ ) adsorbed onto mice from $\mathrm{CH}$ at $26^{\circ} \mathrm{C}$. While it is not possible to make an absolute comparison with the computed results, as we do not have the value of $\phi_{1}$ for this polymer ( $M=$ $9 \times 10^{5}$ ) at the temperature of the experiment (see also earlier remarks concerning PS2), we may make a relative comparison. Thus, also shown in Figure 8 (broken lines) are calculated interaction-distance profiles for $\beta=1.02$ and 1.9 , which correspond to $\Gamma$ values differing by a factor 


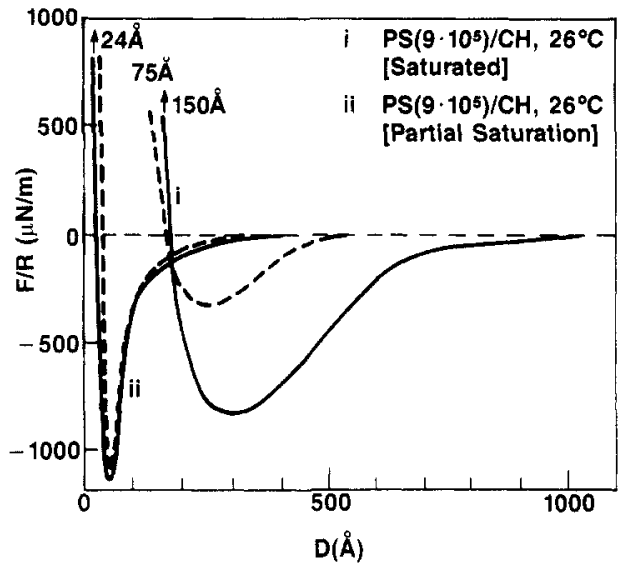

Figure 8. Interaction-distance profiles between mica sheets in polystyrene $\left(M=9 \times 10^{5}\right)$-cyclohexane solution, from ref 5 , following partial adsorption (solid curve ii) and full adsorption (solid curve i). The broken curves are calculated for $\beta=1.90$ and 1.02, which correspond to adsorbance values differing by a factor of 3 ( $\sigma=3$ for both calculated curves), as suggested by the experiment. Energy and distance axes for the calculated curves are scaled to provide a fit at $\beta=1.9$ (broken curve ii).

of 3 (Figure 3) as suggested by the experimental ratio $\Gamma^{\prime} / \Gamma$ (the $D$ and $\gamma$ scales for the calculated curves are in arbitrary units, as $\phi_{1}$ (and hence $\xi$ ) is not known for this system). We note that although the spatial scaling of the calculated profiles is in very fair agreement with experiment (for the same $\Gamma / \Gamma^{\prime}$ ratio), the calculated well depth $\left|\gamma_{\min }\right|$ is predicted to increase considerably more rapidly at lower $D_{\text {eq }}$ than is actually observed. This may possibly be accounted for by the omission of the real volume re. pulsion noted above.

Finally, we note that the lower $\Gamma=\Gamma^{\prime}$ force profile (curve ii, Figure 8) shows an (experimentally determined) exponential decay length for $|\gamma|$ equal to $0.18 R_{\mathrm{g}}$, for $D$ in the range $(0.6-1.2) R_{\mathrm{g}}$, (for $\left.M=9 \times 10^{5}\right)$. We predict, for $M$ $=6 \times 10^{5}$ and $1.04 \lesssim \beta \lesssim 1.24$ an exponential decay length $(0.14-0.18) R_{\mathrm{g}}$ for the range $D=(1.3-2.4) R_{\mathrm{g}}$ (see also inset to Figure 4). While these sets of figures are not strictly comparable, they support the general assertion that the spatial aspects of the interaction are well modeled by this mean-field theory.

\section{Concluding Remarks}

The mean-field model of part $1^{13}$ correctly predicts a nonmonotonic force profile between irreversibly adsorbed layers of polymer below the $\theta$-temperature. The calculations of the present paper show a close qualitative, and fair quantitative, agreement with the experimental results for the system studied (PS/CH/mica). This suggests that the assumption of "local equilibrium" of our model is not too bad when applied to these experiments.

An important feature of our model is that it quantitatively predicts surface-surface interactions between adsorbed polymer layers by using parameters which may be obtained from bulk solution measurements only. These include the detailed shape of the phase-equilibrium diagram (yielding $\phi_{1}$ and $\phi_{\text {eq }}$ for any given temperature $T<$ $\theta)$, the unperturbed radius of gyration of the polymer $R_{\mathrm{g}}$ third virial coefficient $w$, and the mass of polymer adsorbed per unit area of surface incubated in the solution. There are no adjustable parameters; it is not necessary, for example, to specify an arbitrary value for the Flory-Huggins lattice parameter $a$. Possible improvements to the theory would be the inclusion of real volumes and of (experimentally determined) corrections to the Flory-Huggins free energy, arising, for example, from nonconstancy of the polymer-solvent interaction parameter $\chi$.

One prediction currently being tested (with H. Terashima) is whether the onset of the biphasic region is accelerated, in the vicinity of a surface, to volume fractions $\phi<\phi_{1}$; this is one interpretation of the surface adsorbance curve (Figure 3 ) when the translational entropy is retained in the Flory-Huggins free energy (eq II.1).

Finally, we note that in the experiments described in ref 5 on the polystyrene/cyclohexane/mica system $(\theta=34.5$ ${ }^{\circ} \mathrm{C}$ ) a weak attraction persisted even when the temperature was raised to $T \gtrsim \theta$. In contrast, in a slightly different system, polystyrene/cyclopentane $/$ mica $\left(\theta=19.6^{\circ} \mathrm{C}\right)$, monotonic repulsion only is observed at $T \gtrsim \theta$. In a paper currently under preparation we have extended our calculations also to the $\theta$-temperature case: we find that, depending on the magnitude of the third virial coefficient, such behavior is indeed predicted by our model.

Acknowledgment. We thank Professors P. J. Flory and S. F. Edwards for very useful comments and for drawing our attention to ref 23 and 24, and Dr. H. Terashima for communication of adsorbance data prior to publication. We also thank the SERC (UK) for partial support to K.I.

\section{Appendix}

In part 1 of this series, the translational entropy per chain $(\phi / N)$ in $\phi$ in the Flory-Huggins free energy (eq II.1) was neglected when the interfacial energy density $\gamma$ was calculated. If this term is retained, (II.5) becomes

$$
\begin{aligned}
&\left(\gamma-\gamma_{0}\right) /\left(\gamma_{1} \phi_{\mathrm{eq}}\right)=-y_{\mathrm{s}}^{2}+\sigma^{-1} \int_{0}^{\infty}\left[y^{6}-2 y^{4}+\right. \\
&\left.\left(\eta \ln y^{2}+\beta^{2}\right) y^{2}+b+\left(\frac{\mathrm{d} y}{\mathrm{~d} x}\right)^{2}\right] \mathrm{d} x
\end{aligned}
$$

where $\eta=-1 /\left(\ln \phi_{1}\right), \beta^{2}=\left(1+\ln y_{b}{ }^{2}+4 y_{b}{ }^{2}-3 y_{b}{ }^{4}\right) /(\ln$ $\left.\phi_{1}\right)$, and $b=2 y_{\mathrm{b}}{ }^{6}-2 y_{\mathrm{b}}{ }^{4}+\eta y_{\mathrm{b}}{ }^{2}$; all other symbols have the same meaning as in section II. When (A.1) is minimized with respect to $y(x)$, the first integral of the Euler-Lagrange equation yields

$$
(\mathrm{d} y / \mathrm{d} x)^{2}=G(y)-G\left(y_{\mathrm{b}}\right)
$$

where $G(y)=y^{6}-2 y^{4}+\left(\eta \ln y^{2}+\beta^{2}\right) y^{2}+b$ and the $G\left(y_{\mathrm{b}}\right)$ term is included to avoid a logarithmic infinity as $y \rightarrow 0$.

It can be shown that for typical values of $y_{s}>y>y_{b}$ $\left(y_{\mathrm{s}} \sim 1, y_{\mathrm{b}} \sim 10^{-3}\right)$ the terms in $\eta$ and $b$ make a negligible difference to the adsorption profile, but that the redefinition of $\beta$ is significant. Dropping terms of order $y_{b}^{2}$ and higher, we write

$$
\beta^{2}=\frac{1+\ln \left(\phi_{\mathrm{b}} / \phi_{\mathrm{eq}}\right)}{\ln \phi_{1}}
$$

All other equations in section II of part 1 stand unaltered.

Registry No. Polystyrene, 9003-53-6; cyclohexane, 110-82-7.

\section{References and Notes}

(1) Vincent, B. Adv. Colloid Interface Sci. 1974, 4, 193

(2) Vincent, B.; Whittington, S. G. Surface Colloid Sci. 1982, 12, 1.

(3) Klein, J. J. Chem. Soc., Faraday Trans. 1 1983, 79, 99. Nature (London) 1980, 288, 248.

(4) Klein, J.; Luckham, P. F. Nature (London) 1982, 300, 429. Macromolecules 1984, 17, 1041.

(5) Israelachvili, J. N.; Tirrell, M.; Klein, J.; Almog, Y. Macromolecules 1984, 17, 204.

(6) Almog, Y.; Klein, J. J. Colloid Interface Sci. 1985, 106, 33.

(7) Scheutjens, J. M. H. M.; Fleer, G. J. Adv. Colloid Interface Sci. 1982, 16, 341. Macromolecules 1985, 18, 1882.

(8) Rubin, R. J. J. Chem. Phys. 1965, 43, 2392.

(9) Di Marzio, E. A.; Rubin, R. J. J. Chem. Phys, 1971, 55, 4318. 
(10) Cahn, J. J. Chem. Phys. 1977, 66, 3667.

(11) de Gennes, P. G. Macromolecules 1981, 14, 1637.

(12) de Gennes, P. G. Macromolecules 1982, 15, 492.

(13) Klein, J.; Pincus, P. A. Macromolecules 1982, 15, 1129.

(14) Flory, P. J. "Principles of Polymer Chemistry"; Cornell University Press: Ithaca, NY, 1953.

(15) de Gennes, P. G. "Scaling Concepts in Polymer Physics"; Cornell University Press: Ithaca, NY, 1979.

(16) Edwards, S. F. J. Phys. A: Math. Gen. 1975, 8, 1670.

(17) Di Maglio, J. M.; Ober, R.; Paz, L.; Tapin, G.; Pincus, P. A.; Boileau, S. J. Phys. (Paris) 1983, 44, 1035.

(18) McDonnell, M. E., West Virginia University, unpublished data.
(19) Terashima, H.; Klein, J.; Luckham, P. F. In "Adsorption from Solution”; Rochester, C.; Ottewill, R. H., Eds.; Academic Press: London, 1983; p 299.

(20) Brandrup, J.; Immergut, E. H., Eds. "Polymer Handbook"; Wiley: New York, 1975.

(21) Schultz, A. R.; Flory, P. J. J. Am. Chem. Soc. 1952, 74, 4760.

(22) Terashima, H., unpublished data.

(23) Edwards, S. F., private communication. Allen, G.; Burgers, J.; Edwards, S. F.; Walsh, D. J. Proc. B. Soc. London A 1973, 334, 465.

(24) Flory, P. J., private communication. Höcker, H.; Shih, H.; Flory, P. J. Trans. Faraday Soc. 1971, 67, 2275.

\title{
Scaling Analysis of Mechanical and Swelling Properties of Random Polystyrene Networks
}

\author{
R. W. Richards* and N. S. Davidson ${ }^{\dagger}$ \\ Department of Pure and Applied Chemistry, University of Strathclyde, Glasgow G1 1X1, \\ United Kingdom. Received September 17, 1985
}

\begin{abstract}
The equilibrium swelling and shear moduli at swelling equilibrium of randomly cross-linked polystyrene gels have been determined in the excluded-volume limit (good solvent conditions), at the $\theta$ temperature, and in intermediate regimes (below and above the $\theta$ temperature). The results have been compared with data obtained by small-angle neutron scattering and quasi-elastic light-scattering measurements on the same gels and discussed by using current analogies between gels and semidilute solutions. An attempt has been made to correlate both macroscopic and molecular behavior in terms of a fundamental thermodynamic correlation length $\xi_{c}$, the range of the monomer-monomer pair correlation function. Power law behavior for moduli and diffusion coefficients similar to that which has been reported for semidilute solutions was found in some cases, but departures from simple scaling behavior were also evident. Some of these discrepancies have tentatively been attributed to an increasingly loose structure in the gels as the cross-linking density decreases.
\end{abstract}

\section{Introduction}

Polymeric gels comprising a permanent covalently bound network of long-chain molecules much diluted with a low molecular weight solvent display both elastic and viscoelastic properties. ${ }^{1,2}$ They may be regarded either as highly swollen elastomeric solids or as permanently cross-linked solutions of entangled chains. Indeed, when the lifetime of physical entanglements between chains exceeds the time scale of the measurement, the molecular properties of gels and entangled polymer solutions have often been treated as equivalent. ${ }^{3}$

The investigation of macroscopic properties of elastomeric networks such as equilibrium swelling and stressstrain isotherms has been, and continues to be, an area of much effort, being germane to the examination of statistical theories of rubber elasticity. Additionally, the theory of rubber elasticity has a pivotal role in much of polymer science. ${ }^{4-6}$ In recent years there has been renewed interest in gels and entangled polymer solutions stimulated by the development of new theoretical and experimental methods. Techniques such as small-angle neutron scattering, ${ }^{2,-9}$ (SANS) and photon correlation spectroscopy (PCS) ${ }^{10-12}$ have become important as they probe polymer systems on a molecular level, thereby providing data necessary for the rigorous examination of theoretical models. Particular attention has been paid to the analogy between gels and weakly overlapping or semidilute solutions, ${ }^{3}$ utilizing the advances in theoretical descriptions of the latter. In spite of considerable progress in our understanding, there are,

\footnotetext{
* To whom correspondence should be addressed.

${ }^{\dagger}$ Present address: Exxon Research and Engineering Co., Corporate Research Laboratories, Annandale, Ne 08801.
}

however, conflicting experimental data for the global properties of semidilute solutions and no consensus between the theoretical models. Generally the properties in limiting regimes of excluded-volume behavior can be rationalized, but much less well understood is the behavior in situations where the thermodynamic conditions vary over a wide range. Consequently, the situation for true gels is yet more uncertain, as it is compounded by the absence of suitable experimental data.

We report here the results of a series of equilibrium swelling and compressional elastic modulus measurements on randomly cross-linked polystyrene networks covering a range of cross-link density. To study the behavior of the gels under differing thermodynamic conditions, a good (toluene) and a poor diluent (cyclohexane) were chosen as swelling agents. The $\theta$ temperature of polystyrene-cyclohexane systems has been found to be $\sim 35^{\circ} \mathrm{C}$. Consequently the behavior of gels at the excluded-volume limit was studied with polystyrene-toluene gels and at $\theta$ and intermediate conditions (above and below $\theta$ ) with polystyrene-cyclohexane gels. The results are compared with the predictions based on the behavior of $\xi$ values obtained from SANS and PCS measurements on the same gels, which we have previously reported elsewhere, ${ }^{13,14}$ and are discussed in terms of the analogy with semidilute solutions with the aim of obtaining a viewpoint from which both macroscopic and molecular properties are consistent with each other.

\section{Theoretical Background}

The analogy between semidilute polymer solutions and gels swollen by good solvents is embodied in the $c^{*}$ theorem. ${ }^{3}$ Above this critical concentration $c^{*}$, polymer mol- 